\title{
Redescubrimiento de una inscripción latina en el castillo de Castelldefels (Barcelona) *
}

\author{
Alberto López MUllor **
}

El municipio de Castelldefels se encuentra en la comarca del Baix Llobregat, dentro de la provincia de Barcelona, a una veintena de kilómetros al sur de la capital, en un paraje costero caracterizado por su extensa playa y llanura prelitoral, esta última fruto del avance de la linea de costa mediante la desecación de marjaies en fecha relativamente cercana. A mediodia y poniente, el término se halla limitado por el macizo calcáreo de El Garraf, en uno de cuyos contrafuertes, al noroeste del casco urbano, se alza el castillo.

La fortaleza, muy transformada por una intervención de tipo historicista llevada a cabo en 1897, al pasar a manos del banquero barcelonés Manuel Girona, aparece documentada desde el siglo x, como una de las avanzadillas de la marca carolingia frente a la tierra de nadie que la separaba de los dominios musulmanes. Desde el mismo siglo, también se tiene noticias de la iglesia de Santa Maria, conservada actualmente dentro del recinto; templo parroquial hasta su venta al señor Girona, quien la convirtió en oratorio particular restaurándola superficialmente.

Con todo, a raiz de una prospección que realizamos en 1987 con ocasión de elaborarse el catálogo del Patrimonio anexo al Plan General de Ordenación Urbanística del municipio, comprobamos que en las laderas de la elevación donde se encuentra el conjunto, abundaba el material arqueológico de época antigua, pudiéndose suponer la posible presencia de un poblado ibérico y una villa romana.

\footnotetext{
* Una primera versión de este texto fue presentada en forma de comunicación en las I Jornadas Arqueológicas del Baix Llobregat, celebradas en Castelldefels en 1989, cuyas actas permanecen inéditas.

** Servicio de Patrimonio Arquitectónico de la Diputación de Barcelona.
} 


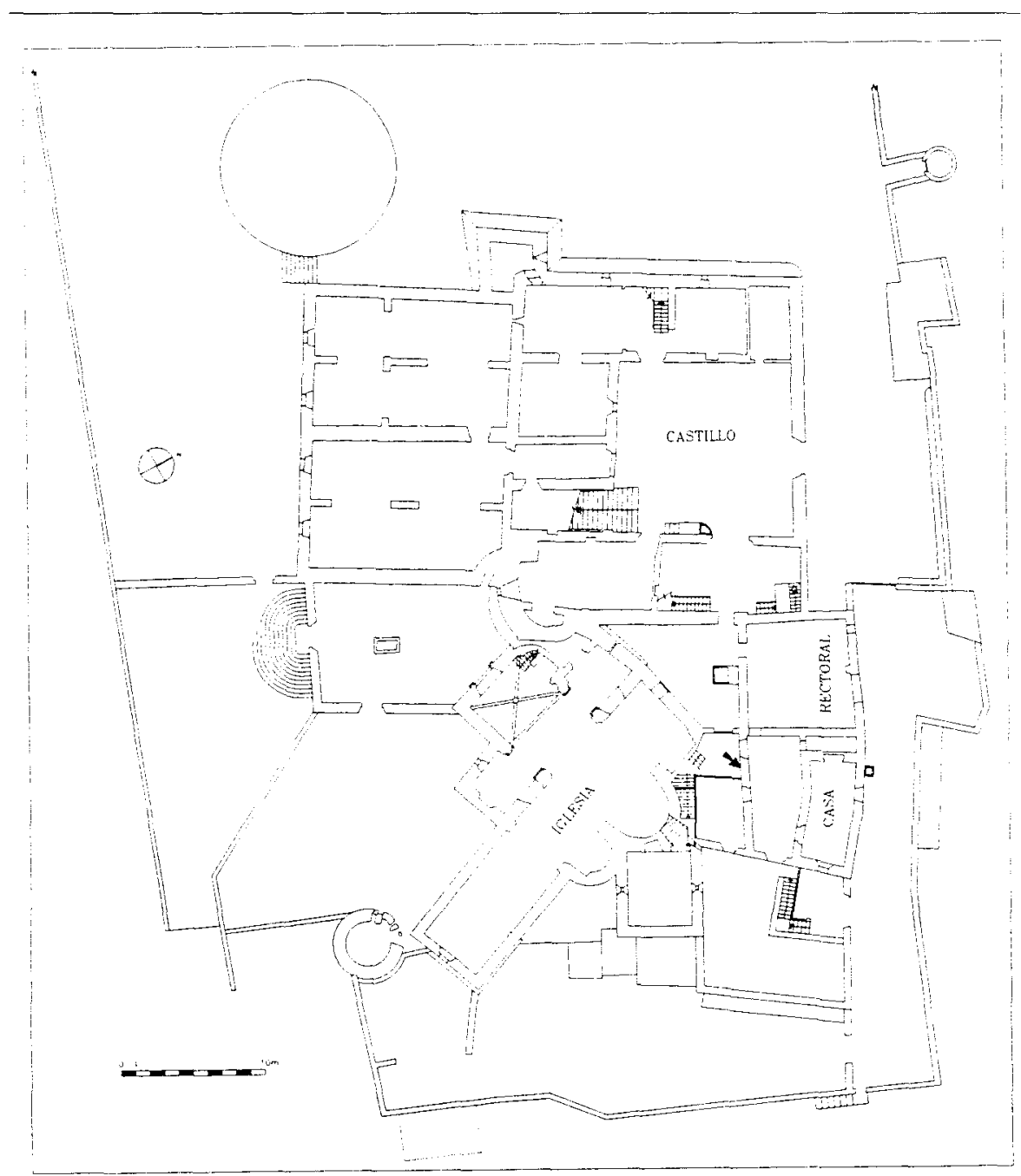

Fig. 1. Planta general del recinto del castillo de Castelldefels antes del inicio de las excavaciones. La flecha indica el lugar donde apareció la inscripción.

En 1989, nuestro Servicio, a resultas de una solicitud municipal, emprendió la restauración de la iglesia de Santa Maria y los edificios de sus alrededores, sacristia y casa rectoral, tomando, además, la responsabilidad de la investigación histórica del resto del recinto (fig. 1), cuya rehabilitación se proyectaba efectuar directamente desde el Ayuntamiento. De acuerdo con nuestro método de trabajo habitual, antes de iniciarse las obras se llevó a cabo un análisis exhaustivo de las fuentes documentales sobre iglesia y castillo, asi como un detenido estudio de historia del arte 
de los mismos. Al tiempo, se emprendió una excavación arqueológica extensiva que, en el momento de redactar estas líneas, se encuentra en su etapa final, y de cuyos resultados esperamos dar cuenta próximamente.

No obstante, hoy nos interesa destacar un descubrimiento o, según veremos, redescubrimiento acaecido pocos dias antes de empezar la actuación sistemática descrita. En febrero de 1989, al establecerse una escuela taller en el castillo, cuyos miembros habrian de colaborar en el mantenimiento del mismo y en las obras de un parque que debía circundarlo, se acondicionaron algunas estancias para utilizarlas durante las clases prácticas. Uno de estos ámbitos se hallaba junto a la cocina de la casa rectoral de la iglesia, sirviendo de distribuidor al acceder a este cuerpo desde el patio interior situado al nordeste del templo (fig. 1). En este espacio, al limpiarse uno de sus tabiques, apareció la cara principal de un cipo o pedestal de piedra, observándose vestigios de una inscripción latina, semioculta debajo de algunas capas de yeso y pintura (figs. 2-5).

Ante estas circunstancias, los trabajos se interrumpieron y la dirección de la escuela nos informó rápidamente del hallazgo. Después de realizar una primera inspección, observamos que efectivamente se trataba de un vestigio antiguo, seguramente bien conservado, decidiéndose extraerlo de la pared para proceder a su restauración y poder conservarlo con las garantias adecuadas. El 1 de marzo, un equipo de la escuela extrajo el bloque, bajo nuestra supervisión y la del arquitecto técnico del Servicio señor José Maria Moreno. Semanas más tarde, la pieza, ya depositada en una estancia del castillo, fue tratada por técnicos del departamento de restauración de la Escuela de Artes y Oficios de la Diputación de BarceIona, dirigidos por la señora Mireia Marquès. Finalmente, fue depositada en un embalaje móvil de madera construido ex professo, conservándose en el propio recinto, a la espera de ocupar en el mismo un lugar preeminente cuando concluyan las obras de restauración.

El cipo es de piedra caliza, tal vez de Santa Tecla, utilizada en otras inscripciones barcelonesas contemporáneas, o bien del vecino macizo de El Garraf, aunque su procedencia exacta habrá de comprobarse mediante los análisis que se hallan en curso. Su estado actual es relativamente bueno, aun cuando presenta algunas erosiones en las aristas. En la cara principal también son visibles diversas grietas, que seguramente ya debió poseer el bloque de antiguo - pues parecen de origen natural-, aunque pueden haberse agudizado con el paso del tiempo.

Las dimensiones de la pieza son las siguientes: $93 \mathrm{~cm}$ de longitud, por $60 \mathrm{~cm}$ de anchura, por $52 \mathrm{~cm}$ de grosor, midiendo el campo epigráfico $78 \mathrm{~cm}$ de largo por $48,5 \mathrm{~cm}$ de ancho. En la cara lateral derecha presenta 


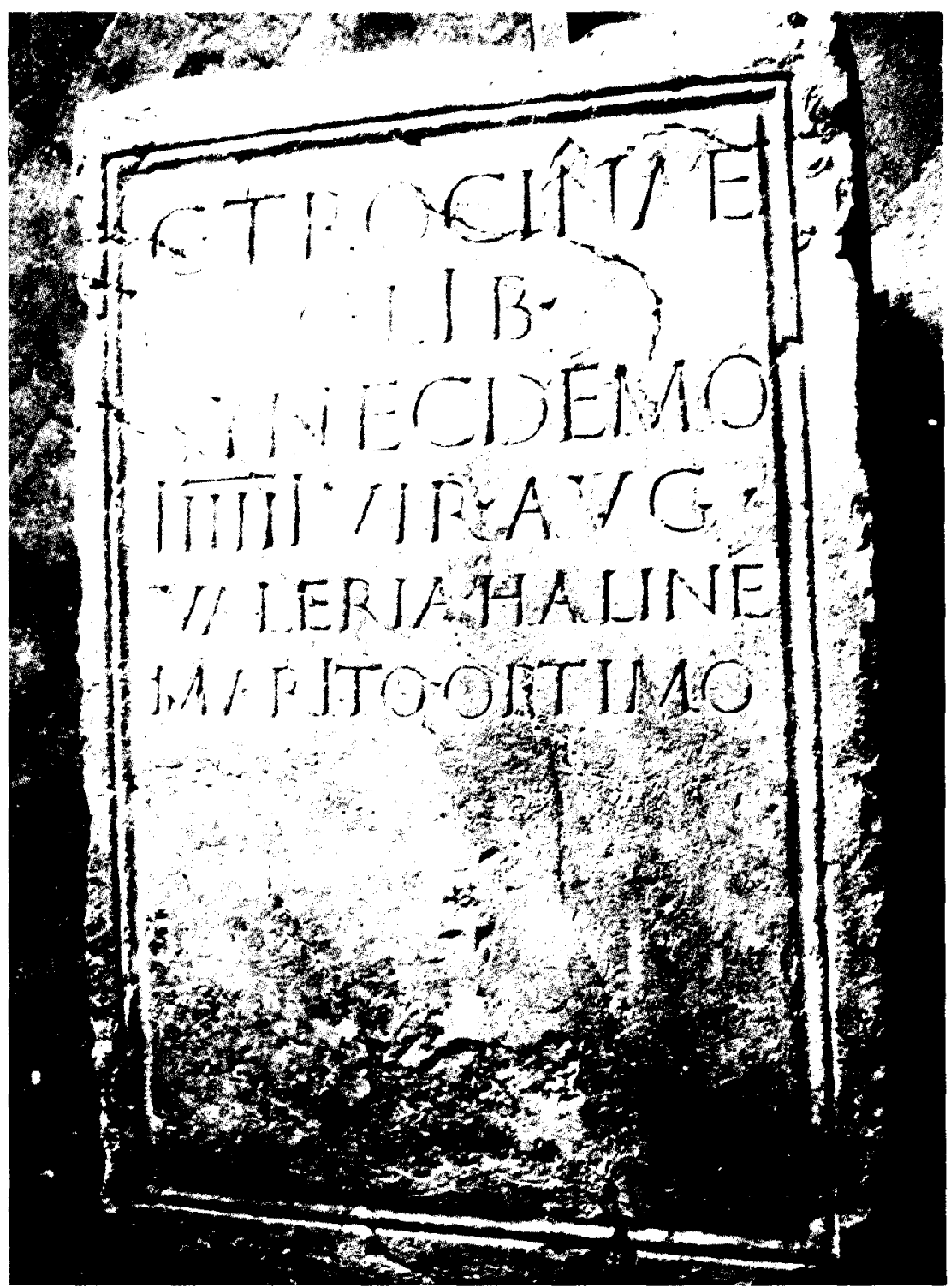

Fig. 2. Cara principal del cipo. 
Redescubrimiento de una inscripción latina en el castillo de ...

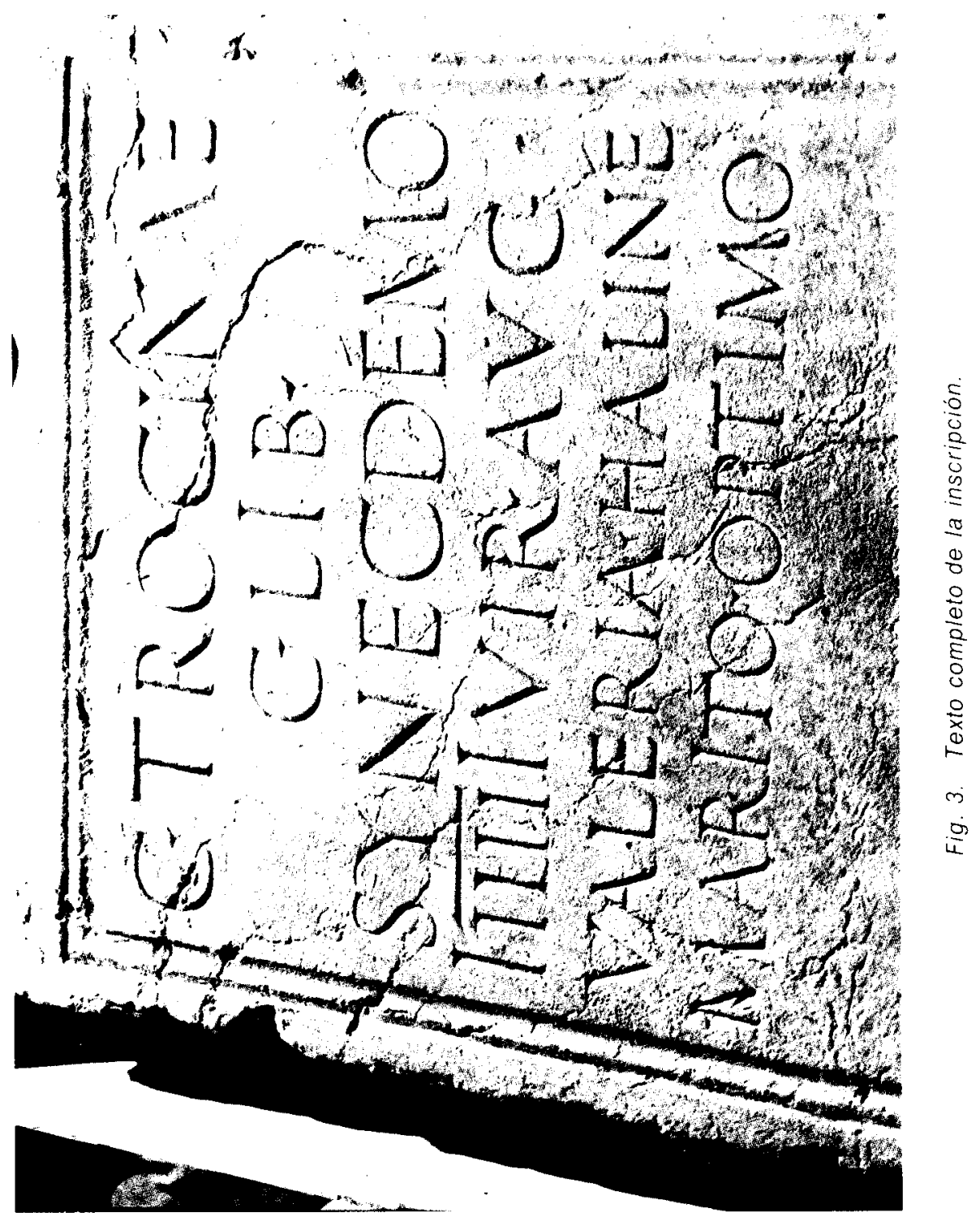




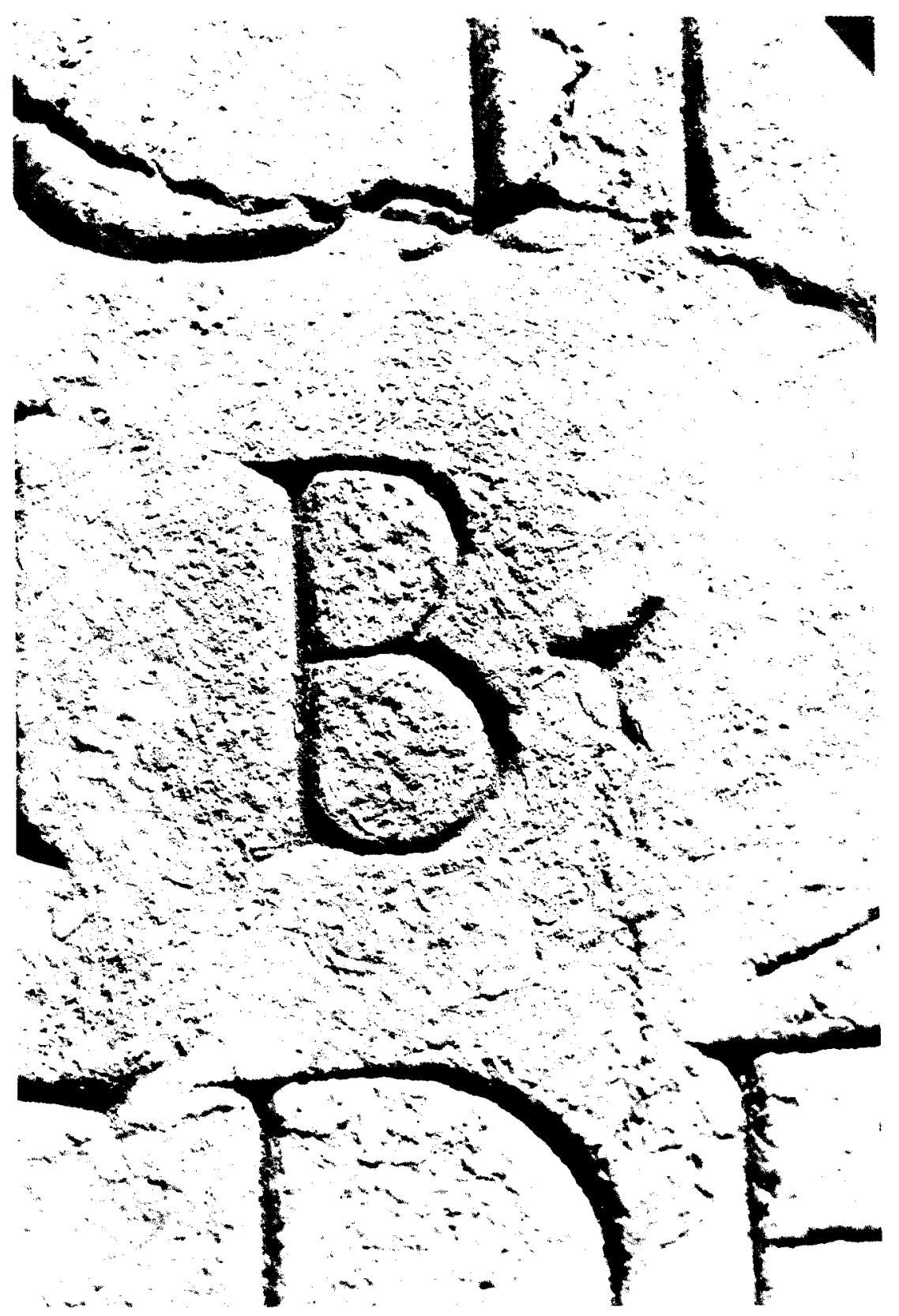

Fig. 4. Detalle de una letra (1.2) acompañada de interpunción. 


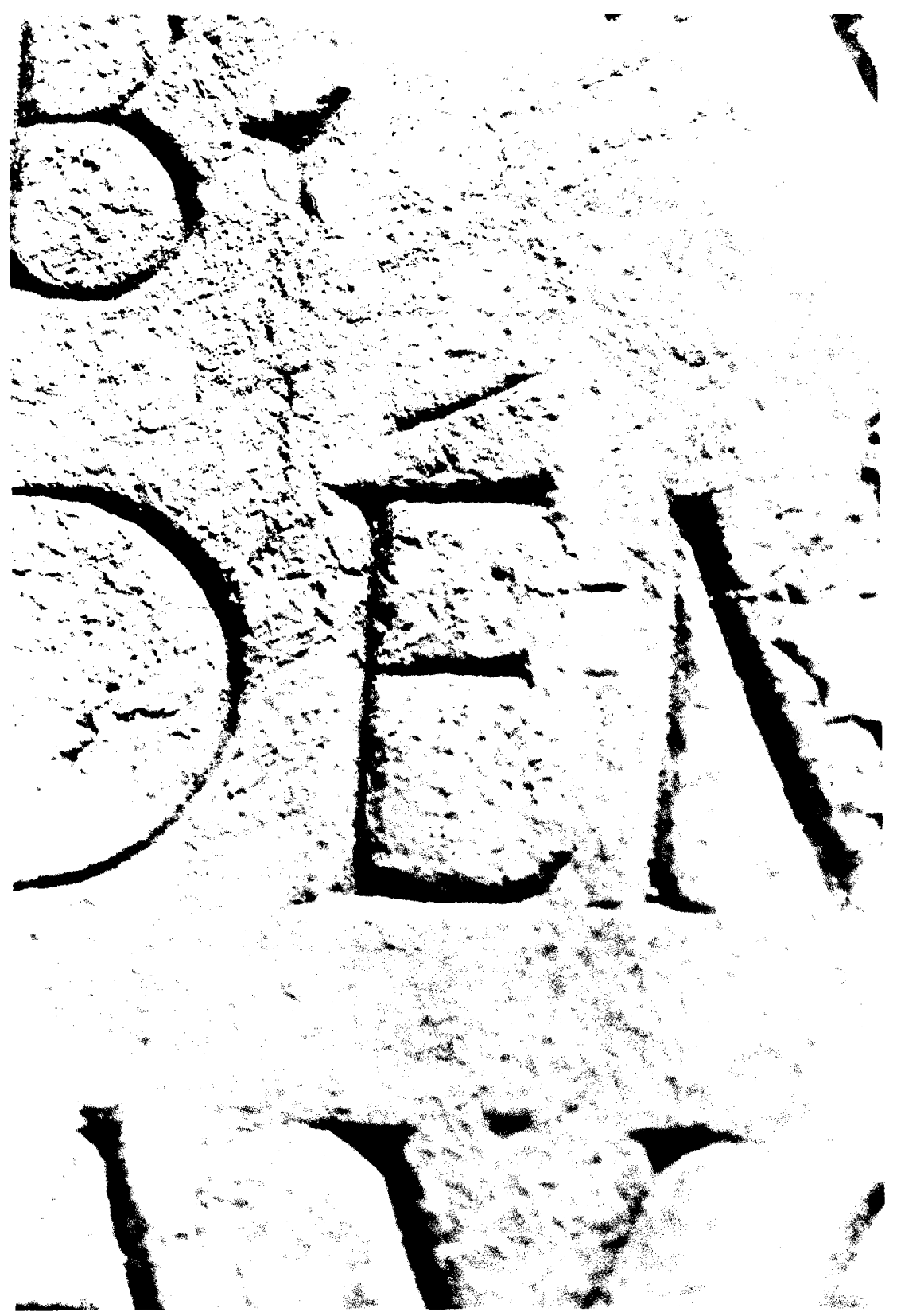

Fig. 5. Detalle de una letra (1.3) acentuada. Los acentos de las dos es pudieran indicar la presencia de etas en la grafia griega. 
dos orificios cilindricos. Todo el elemento está bien trabajado, salvo la cara superior, donde seguramente se apoyaba un coronamiento - quizás una estatua-, y sólo aparece someramente desbastada, presentando irregularidades considerables.

La cara mayor delantera, que hemos denominado principal, se encuentra ribeteada de un baquetón entre cavetos y ostenta la inscripción: C (aio). TROCINAE / C (aii). LIB (erto). / SYNECDÉMO / IIIII VIR (o). AUG (ustali). / VALERIA. HALINÉ / MARITO. OPTIMO (A Cayo Trócina Synecdémo, liberto de Cayo, sevir augustal, (su esposa) Valeria Haliné (se lo dedica) por haber sido un excelente marido).

Para mayores precisiones sobre las dimensiones del texto y las letras, pueden verse el apéndice y las figs. 2-5. Con todo, es claro que se trata de capital cuadrada, relativamente bien trazada, siendo menores los caracteres de 1. 5,6, visiblemente apretados en el lado derecho para no rozar el margen; las interpunciones son triangulares lanceoladas. Además, se observan ies longas en $1,2,6$, y sendos acentos en las es de los cognomina de los personajes citados en 1, 3, 5 .

A pesar de lo que pudiera suponerse, teniendo en cuenta las circunstancias del hallazgo, debe destacarse que esta inscripción no es inédita y su localización de hace dos años fue una especie de resurrección cientifica de la pieza. Un erudito del siglo xviıl la vio en la rectoría de la iglesia de Castelldefels, dibujó un calco y tomó algunas notas. Su manuscrito fue a parar a la Biblioteca de Catalunya (Guia de la Biblioteca Central. Barcelona 1959: 83) y se ha conservado en esta institución hasta nuestros dias. Además de la tarea del personaje dieciochesco, se ha de mencionar otra aportación decisiva para el conocimiento del epigrafe. Se trata de un articulo del profesor $M$. Mayer, donde se estudia el calco mencionado (MAYER 1980).

Ante tales precedentes, nuestra intervención radica esencialmente en comunicar la recuperación del elemento, aportando, en todo caso, algunas precisiones. El calco de la inscripción transmitido literariamente y reproducido por Mayer es bastante correcto. Las dimensiones supuestas por este investigador $(0,90 \times 0,60 \times 0,55 \mathrm{~m})$ son muy próximas a las reales. Igualmente la descripción que hace sobre el tipo de letra, puntuación y otras características es exacta, salvo la omisión de las letras acentuadas, cuya tilde quizá debió pasar desaparecibida al sabio ilustrado.

En cuanto al personaje, recordemos que el nomen Trocina aparece en Tarraco, donde se conocen una Trocina Afrodisia y un Trocina Sirvandus (VIVES 1972: 3554; ALFOLDY 1975; 333, n. ${ }^{\text {os }} 671-672$ ). Además, se sabe de la existencia de Caius Trocina Onesimus y de su heredero Philetus (VIVES 1972, n. ${ }^{\circ}$ 1363). 
En Barcelona aparecen nuevos testimonios de este último individuo: era liberto, alcanzó la magistratura de sevir augustal y seguramente tuvo una relación de clientela hacia L. Licinius Secundus (MANGAS 1971: 380381, 418; Alföldo 1975; 418; Fabre, Mayer, Roda 1984; 168-170, n. ${ }^{\circ} 125$, inscripción en Sant Andreu de Llavaneres). También se menciona su heredero, Trocina Philetus, en este caso acompañado de $C$. Trocina Paramythius, que ostentaba la misma condición (MANGAS 1971; 418; VIVES 1972; 1370-1371). En la propia Barcino, también conocemos un pariente: C. Terentius Onesimus, igualmente servir (MANGAS 1971; 418), quien también aparece en una inscripción de Tortosa (VIVES 1972, $n .{ }^{\circ} 488$ ). No debe olvidarse una mujer llamada Trocina Philumene (Ibidem 4551). Por fin, en Caldes de Montbiu se identifica a otro pariente: C. Trocina Zoticus (FABRE, Mayer, Roda 1984, n. ${ }^{\circ} 38$; Mangas $1989, n .{ }^{\circ} 131$ ).

Como puede verse, la saga de los Trócina es bastante conocida en un territorio de forma triangular, cuyos vértices se encuentran en Sant Andreu de Llavaneres, Caldes de Montbui y Tortosa. A pesar de todo, sobresalen las inscripciones de Barcelona y Tarragona. Parece que estos libertos de origen griego gozaron de influencia, fruto del poder de la gens a que estaban vinculados y acaso también de las relaciones de clientela con L. Licinius Secundus (Trócina Onésimo se declara su amicus), personaje sobradamente conocido, ligado al círculo del emperador Trajano a través de Licinio Sura.

Estas buenas relaciones les podrian haber reportado una innegable consideración social, puesto que la mayoria de los miembros de la familia que conocemos fueron sacerdotes del culto de Augusto, como era el caso de Trócina Synecdémo.

Con estos datos podemos deducir que, aun estando casado nuestro personaje con una mujer de condición u origen servil, acaso originaria de Tarraco (MAYER 1980: 166), quien le dedicó la inscripción (y probablemente el monumento) que le ha hecho pasar a la posteridad, recorrio un cursus municipal sobresaliente, teniendo en cuenta su condición social, y lo más lógico es que fuera propietario rústico.

En este sentido, creemos que el hallazgo de la inscripción en el castillo de Castelldefels no es casual. En primer lugar, debe tenerse en cuenta que transportarla por gusto desde Barcelona durante la edad Moderna no parece demasiado coherente, sobre todo si era para reutilizarla en la construcción de la rectoria de una iglesia. Además, después del siglo XVIII - seguramente ya a finales de esa misma centuria-, el párroco no debia tener el epigrafe en mucha estima, puesto que lo hizo enyesar. Por otra parte, como observa Mayer, falta el permiso de los decuriones para co- 
locar el pedestal en su sitio; y nosotros pensamos que no era menester concederlo ya que el monumento no se instaló en la ciudad.

En segundo lugar, el hecho de haberse encontrado la pieza en un lugar donde existe un yacimiento arqueológico relativamente importante parece la pista más segura. En el momento de localizarse el cipo, las prospecciones ya habian indicado la probable existencia de un poblado y una villa. Ahora, después de dos campañas de excavación, tenemos la certeza de haber localizado ambos tipos de poblamiento, el primero datable, como mínimo, entre mediados del siglo III y finales del siglo I a. C.; el segundo, desde esta última fecha hasta un momento incierto del siglo vı. La villa, además, presenta fases edilicias bien documentadas en los últimos decenios del siglo । a. C., en el siglo $\|$ y en la primera mitad del siglo III.

Por todo ello, creemos que la inscripción se ha descubierto muy cerca del lugar donde estuvo emplazada en la antigüedad, dentro de la villa que Trócina Synecdémo poseyó hacia la primera mitad del siglo II, en la que, por cierto, en vida suya, se realizaron algunas obras.

Para finalizar, añadiremos que esta explotación debió encontrarse dentro del ager de Barcino, pues sospechamos que el macizo del Garraf lo separaba del de Tarraco (LoPEz Mullor 1986; 137; LoPEz MulLoR, FIERRO 1990; 227). Este hecho nos hace pensar que Synecdémo fue sevir en la colonia augústea y que su villa de Castelldefels, entonces a orillas de un pequeño puerto, fue una de las propiedades que dio solvencia a su magistratura.

Tal vez, las excavaciones del yacimiento, que esperamos concluir en el presente año, podrán dar alguna otra precisión sobre el tema. De momento, debemos felicitarnos por el hallazgo de la pieza que, de una manera tan insólita, a vuelto a ver la luz. 


\section{APENDICE}

Dimensiones del epigrafe

- Cara principal: $93,5 \times 60,5 / 61$ (aristas degradadas irregularmente).

-Campo: $78 \times 48,5$.

- Texto: $42 \times 48,5$.

- Molduras laterales 4 (liso) $+0,4$ (1. caveto) $+1,9$ (baquetón) $+0,6$ (2. caveto)

- Lineas, interlíneas, letras y puntuación:

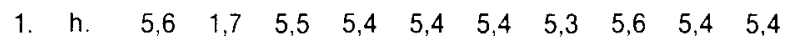

$\begin{array}{lllllllllllllllll}\text { C } & \text { T } & \text { R } & \text { O } & \text { C } & \text { I } & \text { N } & \text { A } & \text { E }\end{array}$

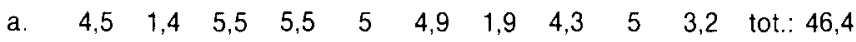

interlinea: 2,3 .

2. h. $4,8 \quad 1,2 \quad 4,8 \quad 5,6 \quad 4,8 \quad 1,6$

$$
\text { C . L L } \quad \text { I } \quad \text { B }
$$

a. $\quad 4,5 \quad 1,2 \quad 3,5 \quad 1,6 \quad 3,2 \quad 1,4 \quad$ tot.: 20

interlinea: $2,2 / 2,4$.

3. h. $\quad 5,3 \quad 5,3 \quad 5,5 \quad 5 \quad 5,2 \quad 5 \quad 5,2 \quad 0,6^{*} \quad 5,2 \quad 5,3$

$$
\begin{array}{llllllllllllllll}
S & Y & N & E & C & D & E^{*} & & X & O
\end{array}
$$

a. $\quad 2,5 \quad 4,4 \quad 5,4 \quad 3,9 \quad 5 \quad 5,1 \quad 3,3 \quad 2^{*} \quad 5,7 \quad 4,4$ tot.: 45,9

dimensiones del tilde sobre la $\mathrm{E}$.

interlinea: $2,3 / 2,5$.

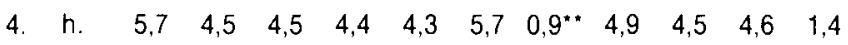
a. $\quad \begin{array}{lllllllllll}1,1 & 1,1 & 1,2 & 1,2 & 1,2 & 1,4 & 8,3 & 4,5 & 1,4 & 4,9 & 1,4\end{array}$
** nexo entre segundo y cuarto numerales.
h. $4,2 \quad 4,4 \quad 4,5$
? (mal conservado)

A $U$ G
a. $\quad \begin{array}{lll}4 & 5 & 4,2\end{array}$
? (id.)
tot.: 42,5

interlinea: $2,4 / 2,5$.

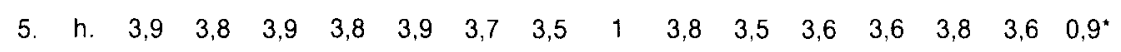
$\begin{array}{llllllllllllll}V & A & L & E & R & \text { I } & A & . & H & A & L & \text { I } & N & E^{*}\end{array}$

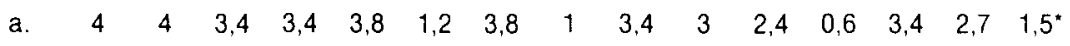
tot: 46,1

* dimensiones del tilde sobre la $E$ interlinea: $2,4 / 2,7$.

$\begin{array}{lllllllllllllll}\text { 6. } & \text { h. } & 4 & 4 & 4 & 4,4 & 3,9 & 3,9 & 1 & 3,9 & 3,8 & 3,7 & 3,7 & 3,7 & 3,8\end{array}$

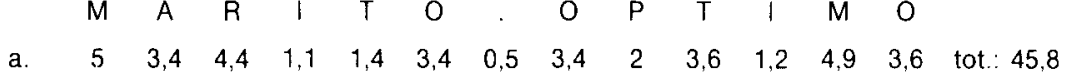




\section{BIBLIOGRAFIA}

Alföloy, G. (1975): Die Römischen Inschriften von Tarraco. Berlin, 2 vols.

FABRE, G.; MAYER, M. y RODA. I. (1984): Inscriptions romaines de Catalogne 1. Barcelone (sauf Barcino). Paris.

López Mullor, A. (1986): "Establiment ibèric i romà de Darró (Establecimiento ibérico y romano de Darró; The Iberian and Roman Establishment at Darró)", Memória 1984, Servei de Catalogació i Conservació de Monuments de la Diputació de Barcelona. Barcelona: 130-142, 198-203, 252-258.

Lopez Mullor, A. y Fierro, J. (1990): “La época romana en Darró (Vilanova i la Geltrú, Barcelona)", Espacio, Tiempo y Forma, Serie I, 3: 203-254.

MANGAS, J. (1971): Esclavos y libertos en la España romana. Salamanca.

Mangas, J. (dir.) (1989): Hispania Epigraphica, 1. Madrid.

MaYer, M. (1980): "Dos inscripciones posiblemente barcelonesas", Rivista di Studi Liguri, XLVI: 158-166.

VIVEs, J. (1972): Inscripciones latinas de la España romana. Antología de 6.000 textos. Barcelona, 2 vols. 